\title{
Gravitational inflaton decay and the hierarchy problem
}

\author{
Yuki Watanabe* \\ Department of Physics, University of Texas, Austin, Texas 78712, USA \\ Eiichiro Komatsu \\ Department of Astronomy, University of Texas, Austin, Texas 78712, USA
}

(Dated: October 29, 2018)

\begin{abstract}
We study implications of the large- $N$ species solution to the hierarchy problem, proposed by G. Dvali, for reheating of the universe after inflation. Dvali's proposal contains additional $N \sim$ $10^{32} Z_{2}$-conserved quantum fields beyond the Standard Model particles with mass $\sim 1 \mathrm{TeV}$, which weaken gravity by a factor of $1 / N$, and thus explain the hierarchy between the Plank scale and the electroweak scale. We show that, in this scenario, the decay rates of inflaton fields through gravitational decay channels are enhanced by a factor of $N$, and thus they decay into $N$ species of the quantum fields very efficiently, in the limit that quantum gravity effects are unimportant for the gravitational decay rate. In order not to violate energy conservation or over-reheat the universe, inflaton mass, vacuum expectation value of inflaton, or non-minimal gravitational coupling should be tightly fine-tuned. Our conclusion holds even when the gravitational decay is prohibited by some symmetry of the theory; the universe may still be over-reheated via annihilation of inflatons, if the number density of inflaton quanta is greater than the critical value.
\end{abstract}

\section{INTRODUCTION}

Gravity is $10^{33}$ times weaker than the weak force. Both forces involve seemingly fundamental constants: Fermi's constant, $G_{\mathrm{F}}=1.17 \times 10^{-5} \mathrm{GeV}^{-2}=(293 \mathrm{GeV})^{-2} \equiv$ $M_{\mathrm{w}}^{-2}$, for the weak force, and Newton's constant, $G=$ $0.671 \times 10^{-38} \mathrm{GeV}^{-2}=\left(1.22 \times 10^{19} \mathrm{GeV}\right)^{-2} \equiv$ $\left(\sqrt{8 \pi} M_{\mathrm{Pl}}\right)^{-2}$ for gravity. This inexplicably large separation between the Planck scale, $M_{\mathrm{Pl}}$, and the weak scale, $M_{\mathrm{w}}$, is the so-called gauge hierarchy problem [1].

Why is it a problem? The radiative corrections to the Higgs boson mass are quadratically divergent and sensitive to the ultraviolet cutoff scale of the particle physics theory, $M_{\mathrm{UV}}$, and thus drive the bare Higgs mass to a very large value, unless $M_{\mathrm{UV}}$ is closer to $M_{\mathrm{w}}$. However, if $M_{\mathrm{UV}}$ is not much higher than $M_{\mathrm{w}}$, one may wonder why $M_{\mathrm{Pl}}$ is much higher than $M_{\mathrm{UV}}$, i.e., gravity is so weak. $^{1}$

Dvali recently proposed a simple but radical solution to the hierarchy problem [3]. He does not use technicolor or supersymmetry, but uses the black hole physics to show that any consistent theory that includes $N Z_{2}$-conserved species of the quantum fields with mass $\Lambda$ must have a value of the Planck mass, which is bounded from below:

$$
M_{\mathrm{Pl}}^{2} \gtrsim N \Lambda^{2}
$$

in a large- $N$ limit. $^{2}$

\footnotetext{
*Electronic address: yuki@astro.as.utexas.edu

${ }^{1}$ In supersymmetric theory, radiative corrections are only logarithmically divergent. If the supersymmetry breaking scale is close to $M_{\mathrm{w}}$, e.g., $\sim 1 \mathrm{TeV}$, it solves the gauge hierarchy problem; however, one still needs to understand the reason why $M_{\mathrm{Pl}}$ and the symmetry breaking scale are so different, i.e., the $\mu$-problem [2].

2 The reference [3] uses the Planck mass, $m_{\mathrm{Pl}} \sim 10^{19} \mathrm{GeV}$, while
}

Therefore, according to Dvali's solution (see also [4]), gravity is weak because there are $N$ species of the quantum fields beyond the Standard Model with mass $\Lambda=O(\mathrm{TeV})$, as well as a discrete $Z_{2}^{N}$-symmetry, with $N \sim 10^{32}$. An example of this scenario is the celebrated large-extra-dimensions solution to the hierarchy problem [5] (see also [6] in the context of String Theory), in which $N \sim 10^{32}$ Kaluza-Klein particles of mass $\sim 1 \mathrm{TeV}$ would appear.

Can we construct a cosmological model that is consistent with Dvali's solution to the hierarchy problem? In particular, can we still construct a successful inflationary scenario, in the presence of such an extremely large number of extra species at the TeV scale?

In this paper, we show that Dvali's proposal is consistent with inflation only when at least one of the followings is tightly fine-tuned: the inflaton mass, $m_{\sigma}$, vacuum expectation value, $\langle\phi\rangle \equiv v$, or non-minimal coupling parameter, $\xi$. While we consider only single field inflation models, our argument can be extended to a multi-field case in a straightforward manner. Here, $\phi$ denotes the inflaton field and its mass is given by the shape of the inflaton potential at the minimum; $\partial^{2} V(\phi) /\left.\partial \phi^{2}\right|_{\phi=v} \equiv m_{\sigma}^{2}$.

Our argument is based exclusively on reheating of the universe after inflation. Even if we do not know details of interactions between the inflaton and matter sector, we do know that there must be decay channels via gravitational interactions, which give the lower bound of the inflaton decay rate.

The existence of a large number of quantum fields with mass, $\Lambda \sim 1 \mathrm{TeV}$, will enhance the decay rate of the inflaton field by $N \sim 10^{32}$. Such a drastic enhancement of the decay rate ought to affect reheating after inflation.

we shall use the reduced Planck mass, $M_{\mathrm{Pl}} \sim 10^{18} \mathrm{GeV}$. 
We shall take a particular point of view when we study implications of Dvali's proposal. In general, one may consider two cut-off scales: one for the particle physics, $M_{\mathrm{UV}}$, and the other for gravity, $M_{\text {grav }}$, which may or may not be the same. In our analysis, we shall assume $M_{\mathrm{UV}} \sim \Lambda$ and $M_{\text {grav }} \gg \Lambda$. This assumption allows us to analyze the gravitational inflaton decay in the semiclassical limit, without worrying about quantum gravitational effects.

The paper is organized as follows. In Sec. [II we discuss generic properties of the enhanced decay of inflaton. We especially consider $f(\phi) R$ gravity as an example. In Sec. III we discuss specific models with $V(\phi)=$ $\frac{\lambda}{4}\left(\phi^{2}-v^{2}\right)^{2}$. In Sec. IV we study reheating via pair annihilation of inflatons and compare that to the gravitational decay. For concreteness, we shall consider single-field inflation models, and assume $\Lambda \sim 1 \mathrm{TeV}$ and $N \sim 10^{32}$ throughout this paper unless stated otherwise. We work with the metric signature $(-+++)$.

\section{ENHANCED DECAY OF INFLATON}

In theories with non-minimal couplings between the Ricci curvature and scalar fields, e.g., supergravity, $\mathcal{R}^{2}$ gravity, scalar-tensor gravity, and higher dimensional gravity theories, inflaton fields can decay via gravitational effects. by

The perturbative decay rate, $\Gamma_{\text {grav }}$, is typically given

$$
\Gamma_{\text {grav }} \sim N C \frac{m_{\sigma}^{3}}{M_{\mathrm{Pl}}^{2}}
$$

where $C$ is a model-dependent fudge factor. Although the gravitational decay rate is suppressed by the Planck scale, the large number of species, $N$, would compensate it. One usually takes $N \sim 10^{2}-10^{3}$ and $N C \sim \mathcal{O}(1)$.

In Dvali's scenario, $N \sim 10^{32}$, and $M_{\mathrm{Pl}}^{2}$ is bounded from below by Eq. (11). The decay rate is therefore bounded from above as

$$
\Gamma_{\text {grav }} \lesssim C \frac{m_{\sigma}^{3}}{\Lambda^{2}} .
$$

When Dvali's bound is saturated, the decay proceeds very fast and produces radiation ${ }^{3}$ and entropy in the universe efficiently.

But, what if the decay is too efficient, and too much radiation is produced? This is the argument that we shall use throughout this paper.

The most conservative, and model-independent constraint on Dvali's proposal can be obtained by the following argument: at any epoch during or after inflation,

\footnotetext{
3 The "radiation" may contain both visible and hidden sectors.
}

the energy density of the universe must not exceed the Planck energy density.

If the energy density of inflaton during inflation is less than the Planck energy density, $\rho_{\text {inf }}<M_{\mathrm{Pl}}^{4}$, energy conservation demands that the energy density of radiation must also satisfy $\rho_{\text {rad }}<\rho_{\text {inf }}<M_{\mathrm{Pl}}^{4}$.

The expansion rate of the universe during reheating roughly equals the total decay rate of inflaton, $H\left(t_{\mathrm{rh}}\right) \sim$ $\Gamma_{\text {tot }} \gtrsim \Gamma_{\text {grav }}$. From the Friedmann equation and Eq. (2), we get $\left(\Gamma_{\text {grav }} M_{\mathrm{Pl}}\right)^{2} \sim\left(N C m_{\sigma}^{3} / M_{\mathrm{Pl}}\right)^{2} \lesssim \rho_{\text {rad }}<M_{\mathrm{Pl}}^{4}$. Solving this inequality for inflaton mass, we get an upper limit on the inflaton mass,

$$
m_{\sigma}<10^{8} \mathrm{GeV}\left(\frac{10^{30}}{N C}\right)^{1 / 3} .
$$

This constraint is not very interesting in the conventional scenario in which $N C \sim \mathcal{O}(1)$, e.g., $m_{\sigma}<$ $10^{18} \mathrm{GeV}$.

However, in a large- $N$ limit, say $N C \sim 10^{30}$, the constraint becomes very tight: $m_{\sigma}<10^{8} \mathrm{GeV} \sim 10^{-10} M_{\mathrm{Pl}}$, which is significantly tighter than the usual fine-tuning of the inflaton mass, $m_{\sigma} \sim 10^{-6} M_{\mathrm{Pl}}$, for a successful chaotic inflation model with $V(\phi)=m_{\sigma}^{2} \phi^{2} / 2$. Therefore, Dvali's proposal is consistent only when the inflaton is very light, significantly lighter than the conventional case. While $m_{\sigma}$ is fined-tuned with respect to $M_{\mathrm{Pl}}$ (or $M_{\text {grav }}$ ), it would be natural to have $m_{\sigma} \sim M_{\mathrm{UV}} \sim O(\mathrm{TeV})$ whose value is consistent with Eq. (4).

A more powerful constraint comes from the expansion rate of the universe during inflation, $H_{\mathrm{inf}}$. The energy density of inflaton during inflation is related to $H_{\text {inf }}$ via the Friedmann equation, $\rho_{\mathrm{inf}}=3 M_{\mathrm{Pl}}^{2} H_{\mathrm{inf}}^{2}$. Energy conservation then demands that the energy density of radiation must satisfy $\rho_{\text {rad }}<\rho_{\text {inf }}=3 M_{\mathrm{Pl}}^{2} H_{\text {inf }}^{2}$, which yields a bound on the gravitational decay rate, $\Gamma_{\text {grav }}<\sqrt{3} H_{\text {inf }}$.

This bound may also be found as follows: since the expansion rate decreases as inflation ends, the expansion rate during inflation, $H_{\mathrm{inf}}$, is greater than that during reheating: $H_{\mathrm{inf}}>H\left(t_{\mathrm{rh}}\right) \sim \Gamma_{\text {tot }} \gtrsim \Gamma_{\text {grav }}$. We can use this inequality to constrain $\Gamma_{\text {grav }}$, if we know what $H_{\text {inf }}$ is.

How do we constrain $H_{\text {inf }}$ observationally? The amplitude of primordial gravity waves is related to the expansion rate of the universe during inflation as

$$
H_{\mathrm{inf}}^{2} \simeq \frac{\pi^{2} M_{\mathrm{Pl}}^{2} \Delta_{h}^{2}(k)}{2}=\frac{\pi^{2} M_{\mathrm{Pl}}^{2} r \Delta_{\mathcal{R}}^{2}(k)}{2},
$$

where $\Delta_{h}^{2}(k)$ and $\Delta_{\mathcal{R}}^{2}(k)$ are the dimensionless power spectrum of tensor and curvature perturbations, respectively [7]. The current observational constraint on the tensor-scalar ratio, $r \equiv \Delta_{h}^{2}(k) / \Delta_{\mathcal{R}}^{2}(k)$, is $r \lesssim 1$, and the curvature perturbation is of order $\Delta_{\mathcal{R}}^{2}(k) \sim 2 \times 10^{-9}$ [8, 9].

Combining these with Eq. (2), we get a tighter constraint,

$$
m_{\sigma}<10^{7} \mathrm{GeV}\left(\frac{10^{30}}{N C}\right)^{1 / 3}\left(\frac{r \Delta_{\mathcal{R}}^{2}}{2 \times 10^{-9}}\right)^{1 / 6}
$$


Note that this limit is also fairly model-independent, and makes the fine-tuning of the inflaton mass even tighter.

As this constraint is insensitive to the precise value of $r$ or $\Delta_{\mathcal{R}}^{2}$, the future observations of the B-mode polarization of the cosmic microwave background, which would reach $r \sim \mathcal{O}\left(10^{-2}\right)$, will not improve the constraint significantly.

One can obtain even tighter constraints by considering the following limit on the reheating temperature,

$$
1 \mathrm{MeV} \lesssim T_{\mathrm{rh}} \lesssim 10^{8} \mathrm{GeV}
$$

where the lower bound comes from the successful primordial nucleosynthesis and the upper bound comes from the requirement that thermal overproduction of gravitino/moduli be avoided [10, 11, 12]. While the lower bound on the temperature must be satisfied for any mod$\mathrm{els}^{4}$, the upper bound is a model-dependent limit. Therefore, this limit is not as generic as the previous two limits. Nevertheless, the resulting constraint on the inflaton mass is the strongest, as we shall show below.

If the gravitational decay is a dominant process $\left(\Gamma_{\text {tot }} \sim\right.$ $\left.\Gamma_{\text {grav }}\right)$, we get $N T_{\text {rh }}^{4} \sim\left(\Gamma_{\text {tot }} M_{\mathrm{Pl}}\right)^{2} \sim\left(N C m_{\sigma}^{3} / M_{\mathrm{Pl}}\right)^{2}$, where we have used the Friedmann equation and Eq.(2); thus, the reheat temperature is given by

$$
T_{\mathrm{rh}} \sim N^{1 / 4} \sqrt{\frac{C m_{\sigma}^{3}}{M_{\mathrm{Pl}}}},
$$

and the above limit on $T_{\text {rh }}$ from the nucleosynthesis and gravitino/moduli problem yields

$$
0.1 \mathrm{GeV}<m_{\sigma}\left(\frac{N C^{2}}{10^{30}}\right)^{1 / 6}<10^{6.3} \mathrm{GeV}
$$

which can be tighter than Eqs. (4) and (6), depending on C.

Even if the decay process is dominated by nongravitational ones (i.e. direct interactions), the upper bound is still valid, as the gravitational decay channel gives the minimal decay rate. The lower bound is a necessary condition to reheat the universe after inflation mainly by gravitational decay of inflaton.

At the earlier stage of reheating, there may be nonperturbative decay of inflaton via preheating, depending on the magnitude of direct interactions. Our argument is valid even after preheating, if any, as the gravitational decay channel gives the minimal decay rate in any case.

Here, we have used a simple, but rather crude, argument to make the main point of this paper. There is

\footnotetext{
${ }^{4}$ Here, we assume that $N$-species fields are unharmful and cascade into radiation in the visible sector, or stable dark matter particles, eventually. If $N$-species fields are long-lived, the reheat temperature is roughly given by $T_{\mathrm{rh}} \sim \sqrt{M_{\mathrm{Pl}}}\left(\Gamma_{\sigma}^{-1}+\Gamma_{N}^{-1}\right)^{-1 / 2}$, where $\Gamma_{\sigma}$ is the total decay rate of inflaton and $\Gamma_{N}$ is that of $N$-species fields. Long-lived, but unstable, $N$-species fields might cause problems, in a way similar to the late decay of moduli [11].
}

still one unknown quantity, $C$, which depends on specific models. How do we determine $C$ ? We shall present more concrete models in the following sections.

\section{A. Decay induced by $f(\phi) R$ gravity}

In this section we use $C$ that we have derived in [13].

Almost all candidate theories of fundamental physics that involve some compactification of the extra spatial dimensions are expected to yield $f(\phi) R$ term, instead of the Einstein-Hilbert term, in the action, the form of $f(\phi)$ depending on models.

The gravitational decay rate of inflaton into all the species that could have existed at the reheating epoch is then given by $C=\left[F_{1}(v)\right]^{2} /\left(128 \pi M_{\mathrm{Pl}}^{2}\right)[13]$, or

$$
\Gamma_{\text {tot }} \simeq N \frac{\left[F_{1}(v)\right]^{2}}{128 \pi M_{\mathrm{Pl}}^{2}} \frac{m_{\sigma}^{3}}{M_{\mathrm{Pl}}^{2}},
$$

where $F_{1}(v) \equiv\left|f^{\prime}(v)\right|\left[1+\frac{3}{2}\left(f^{\prime}(v) / M_{\mathrm{Pl}}\right)^{2}\right]^{-1 / 2}$, $f^{\prime}(v) \equiv \partial f /\left.\partial \phi\right|_{\phi=v}$, and $v \equiv\langle\phi\rangle$ is the vacuum expectation value of $\phi$. Here, $N$-species fields are scalars that are minimally coupled to gravity with a single mass scale, $\Lambda$, and $\Lambda \ll m_{\sigma} .{ }^{5}$ Of course, all the fields do not need to have exactly the same mass, and our argument still applies when they have a moderate mass spectrum.

In this model the inequality Eq.(4) becomes

$$
m_{\sigma}<10^{8} \mathrm{GeV}\left(\frac{10^{32}}{N}\right)^{1 / 3}\left(\frac{M_{\mathrm{Pl}}}{F_{1}(v)}\right)^{2 / 3} .
$$

To make the constraint on $m_{\sigma}$ slightly more general, let us parametrize $N$ in terms of $\alpha$ as $\alpha \equiv N \Lambda^{2} / M_{\mathrm{Pl}}^{2} \lesssim$ 1. In order to solve the hierarchy problem with Dvali's argument, $\alpha \sim 1$ is required. Note that the minimum of this parameter is given by $\alpha_{\mathrm{grav}}=\Lambda^{2} / M_{\mathrm{Pl}}^{2}$, which represents weakness of gravity for particles with mass of $\Lambda$.

Assuming a typical value of $\left|f^{\prime}(v)\right| \sim M_{\mathrm{Pl}}$, we find that the inflaton mass must be tuned to be smaller than $10^{8} \alpha^{-1 / 3} \mathrm{GeV}$. This constraint is most stringent when the hierarchy problem is solved (i.e. $\alpha \sim 1$ ).

One may reverse the argument by taking the inflaton mass to be a typical value of chaotic inflation, $m_{\sigma} \sim 10^{12} \mathrm{GeV}$, which limits $f^{\prime}(v)$ as $F_{1}(v) / M_{\mathrm{Pl}} \sim$ $\left|f^{\prime}(v)\right| / M_{\mathrm{Pl}}<10^{-6} \alpha^{-1 / 2}$, i.e., $f^{\prime}(v)$ must be fine-tuned.

The expansion rate during inflation gives a stronger constraint [Eq. [6] ]:

$m_{\sigma}<10^{7} \mathrm{GeV}\left(\frac{10^{32}}{N}\right)^{1 / 3}\left(\frac{M_{\mathrm{Pl}}}{F_{1}(v)}\right)^{2 / 3}\left(\frac{r \Delta_{\mathcal{R}}^{2}}{2 \times 10^{-9}}\right)^{1 / 6}$.

\footnotetext{
${ }^{5}$ While we consider scalar matter (bosons) only, one can calculate $C$ for fermions as well. However, the gravitational decay channel to those light (compared to inflaton) fermions is suppressed by their mass, as massless fermions are conformally coupled to gravity [13].
} 
For $\left|f^{\prime}(v)\right| \sim M_{\mathrm{Pl}}, m_{\sigma}<10^{7} \alpha^{-1 / 3} \mathrm{GeV}$. For $m_{\sigma} \sim$ $10^{12} \mathrm{GeV}, F_{1}(v) / M_{\mathrm{Pl}} \sim\left|f^{\prime}(v)\right| / M_{\mathrm{Pl}}<10^{-7.5} \alpha^{-1 / 2}$.

The limit on the reheating temperature [Eq. (7)] yields even stronger constraint [Eq. (8)]:

$$
0.3 \mathrm{GeV}<m_{\sigma}\left(\frac{N}{10^{32}}\right)^{1 / 6}\left(\frac{F_{1}(v)}{M_{\mathrm{Pl}}}\right)^{2 / 3}<10^{6.8} \mathrm{GeV}
$$

For $\left|f^{\prime}(v)\right| \sim M_{\mathrm{Pl}}$, the upper limit on the mass is $m_{\sigma}<$ $10^{6.8} \alpha^{-1 / 6} \mathrm{GeV}$. For $m_{\sigma} \sim 10^{12} \mathrm{GeV}, F_{1}(v) / M_{\mathrm{Pl}} \sim$ $\left|f^{\prime}(v)\right| / M_{\mathrm{Pl}}<10^{-8} \alpha^{-1 / 4}$. $^{6}$

In summary, we have confirmed that, using a physically motivated form of $C$, Dvali's large- $N$ species solution to the hierarchy problem demands tight fine-tuning of $m_{\sigma}$ or $f^{\prime}(v)$. But, how bad are these fine-tunings?

\section{WORKED EXAMPLE: GINZBURG-LANDAU POTENTIAL}

The precise values of $m_{\sigma}$ and $f^{\prime}(v)$ depend on models. We shall study this point in further detail, by using a specific model given by the following action:

$$
\begin{aligned}
\mathcal{L}= & \sqrt{-g}\left[\frac{1}{2} f(\phi) R-\frac{1}{2} \partial_{\mu} \phi \partial^{\mu} \phi-V(\phi)\right]+\mathcal{L}_{\text {matter }} \\
& f(\phi)=M_{\mathrm{Pl}}^{2}+\xi\left(\phi^{2}-v^{2}\right) \\
& V(\phi)=\frac{\lambda}{4}\left(\phi^{2}-v^{2}\right)^{2}
\end{aligned}
$$

where the form of $f(\phi)$ is a popular non-minimal coupling $^{7}$ with the condition, $f(v)=M_{\mathrm{P} 1}^{2}$, that recovers General Relativity after inflation [13, 14, 15].

The form of $V(\phi)$ is also a popular Ginzburg-Landautype potential. Therefore, our example is not exotic or peculiar; on the contrary, this is one of the best studied case, and therefore we hope that this example helps ones understand better how much fine-tuning is required by Dvali's solution to the hierarchy problem.

We now consider what happens when inflaton leaves the slow-roll regime, and begins to oscillate around its potential minimum. The inflaton quanta from the oscillations decay into relativistic $N$ species at least perturbatively and gravitationally. The inflaton mass is given by the curvature of potential around the minimum, $m_{\sigma}^{2} \equiv \partial^{2} V(\phi) /\left.\partial \phi^{2}\right|_{\phi=v}=2 \lambda v^{2}$.

We use the most conservative (probably overly conservative) limit on the radiation energy, $\rho_{\text {rad }}<M_{\mathrm{Pl}}^{4}$ [Eq. (10)], to find a limit on $\lambda$ and $|\xi|$ as

$\lambda<\frac{10^{-20}}{|\xi|^{4 / 3}}\left(\frac{10^{32}}{N}\right)^{2 / 3}\left(\frac{M_{\mathrm{Pl}}}{v}\right)^{10 / 3}\left(1+6 \xi^{2} \frac{v^{2}}{M_{\mathrm{Pl}}^{2}}\right)^{2 / 3}$

\footnotetext{
6 The upper limit is identical to Eq. (18) in [13], when $\alpha \sim 10^{-32}$.

7 In our notation, the conformal coupling corresponds to $\xi=-1 / 6$.
}

which is extremely tight, compared to the existing constraints from inflation [15, 16, 17, 18, 19, 20, 21]. In fact, this limit excludes most of the parameter space allowed by the WMAP 3-yr data. One can find even stronger constraints by considering the expansion rate during inflation, or the limits on the reheating temperature from the gravitino/moduli problem.

It follows from Eq. (12) that it is difficult to avoid finetuning of one parameter without fine-tuning the other parameters. Either $\lambda, \xi$, or $v / M_{\mathrm{Pl}}$, or perhaps all of them, need to be fine-tuned for the large- $N$ species solution to the hierarchy problem to be consistent with inflationary cosmology.

\section{REHEATING BY PAIR ANNIHILATION OF INFLATONS}

So far, we have studied implications of the gravitational decay of inflatons enhanced by the existence of large- $N$ species. In deriving Eq. (2), we assume that inflatons decay into $N$-species fields via an effective trilinear vertex, $\mathcal{L}_{\text {int }} \propto \sigma \chi \chi$, which couples inflaton quanta, $\sigma$, to a pair of $N$ species, $\chi$. However, what if such a coupling is forbidden by the symmetry of the inflaton field? Are there any other channels to reheat the universe after inflation?

In theories of $f(\phi) R$ gravity, the effective interaction Lagrangian is given by the Taylor series expansion of $f(\phi)$ around the vacuum expectation value of $\phi, \phi=v+\sigma$, where $\sigma$ is the inflaton quanta. We find [13]

$$
\begin{aligned}
\frac{\mathcal{L}_{\text {int }}}{\sqrt{-g}}= & \frac{f^{\prime}(v)}{M_{\mathrm{Pl}}^{2}}\left[\sigma U(\chi)-\left(\partial_{\mu} \sigma\right) G^{\mu}(\chi)\right] \\
& +\frac{f^{\prime \prime}(v)}{2 M_{\mathrm{Pl}}^{2}}\left[\sigma^{2} U(\chi)-2 \sigma\left(\partial_{\mu} \sigma\right) G^{\mu}(\chi)\right] \\
& +\frac{\left[f^{\prime}(v)\right]^{2}}{2 M_{\mathrm{Pl}}^{4}}\left[2 \sigma\left(\partial_{\mu} \sigma\right) G^{\mu}(\chi)-\left(\partial_{\mu} \sigma\right)\left(\partial_{\nu} \sigma\right) H^{\mu \nu}(\chi)\right] \\
& +\frac{f^{\prime \prime \prime}(v)}{6 M_{\mathrm{Pl}}^{2}}\left[\sigma^{3} U(\chi)+\ldots\right]+\ldots
\end{aligned}
$$

where $U(\chi)$ is the scalar field potential, e.g., $U(\chi)=$ $m_{\chi}^{2} \chi^{2} / 2+\lambda \chi^{4} / 4+\ldots$, and the other functions are given by

$$
\begin{aligned}
G^{\mu} & =\frac{1}{2} g^{\mu \alpha} \chi\left(\partial_{\alpha} \chi\right), \\
H^{\mu \nu} & =\frac{1}{4} g^{\mu \nu} \chi^{2} .
\end{aligned}
$$

Note that one can also derive the interaction Lagrangian for fermions systematically in a similar manner.

The first terms in $\mathcal{L}_{\text {int }}$ that are proportional to $f^{\prime}(v)$ yield the decay of $\sigma$ with the rate given by Eq. (9), whereas those proportional to $f^{\prime \prime}(v)$ and $\left[f^{\prime}(v)\right]^{2}$ yield the pair annihilation.

Now, let us imagine that the first derivative of $f(\phi)$ vanishes at the vacuum expectation value, $f^{\prime}(v)=0$, 
which will shut off the decay channel. We also assume that $\chi$ are massive free fields, $U(\chi)=m_{\chi}^{2} \chi^{2} / 2$. The interaction Lagrangian at the lowest order in $\sigma$ for this case is given by

$$
\frac{\mathcal{L}_{\text {int }}}{\sqrt{-g}}=\frac{f^{\prime \prime}(v)}{2 M_{\mathrm{Pl}}^{2}}\left[\frac{1}{2} m_{\chi}^{2} \sigma^{2} \chi^{2}-g^{\mu \nu} \sigma\left(\partial_{\mu} \sigma\right) \chi\left(\partial_{\nu} \chi\right)\right],
$$

which may also be written in the form of $\hat{g}^{2} \hat{\sigma}^{2} \chi^{2}$ (after some integration by parts and the use of equation of motion, $\square \chi=\partial U / \partial \chi)$, where $\hat{g}^{2}=$ $f^{\prime \prime}(v)\left[1+\frac{3}{2}\left(f^{\prime}(v) / M_{\mathrm{Pl}}\right)^{2}\right]^{-1}\left(m_{\chi}^{2}+s / 2\right) /\left(4 M_{\mathrm{Pl}}^{2}\right)$ and $s \equiv$ $-g_{\mu \nu}\left(q_{1}^{\mu}+q_{2}^{\mu}\right)\left(q_{1}^{\nu}+q_{2}^{\nu}\right)$ is the square of the total initial 4-momentum of incoming inflaton quanta. We must cannonically normalize inflaton quanta as $\hat{\sigma} \equiv$ $\sigma \sqrt{1+\frac{3}{2}\left(f^{\prime}(v) / M_{\mathrm{Pl}}\right)^{2}}$. We calculate the annihilation cross section from this interaction Lagrangian as

$$
\sigma_{\mathrm{ann}}=N \frac{\left[F_{2}(v)\right]^{2}}{32 \pi M_{\mathrm{Pl}}^{4}} \frac{1}{s}\left(m_{\chi}^{2}+\frac{s}{2}\right)^{2} \sqrt{\frac{s-4 m_{\chi}^{2}}{s-4 m_{\sigma}^{2}}},
$$

where $F_{2}(v) \equiv\left|f^{\prime \prime}(v)\right|\left[1+\frac{3}{2}\left(f^{\prime}(v) / M_{\mathrm{Pl}}\right)^{2}\right]^{-1}$.

The annihilation rate of inflatons is then given by

$$
\begin{aligned}
\Gamma_{\mathrm{ann}} & \equiv n_{\sigma}\left\langle\sigma_{\mathrm{ann}} v_{\mathrm{rel}}\right\rangle \\
& \simeq N \frac{3\left[F_{2}(v)\right]^{2} H_{\mathrm{rh}}^{2}\langle s\rangle}{64 \pi M_{\mathrm{Pl}}^{2} m_{\sigma}} \geq N \frac{3\left[F_{2}(v)\right]^{2} H_{\mathrm{rh}}^{2} m_{\sigma}}{16 \pi M_{\mathrm{Pl}}^{2}}(17)
\end{aligned}
$$

where $n_{\sigma}=\rho_{\sigma} / m_{\sigma} \simeq 3 M_{\mathrm{Pl}}^{2} H_{\mathrm{rh}}^{2} / m_{\sigma}$ is the number density of inflaton quanta. In deriving Eq. (17) we have assumed $m_{\chi} \ll m_{\sigma}$. The relative velocity, $v_{\text {rel }}$, is given by $v_{\text {rel }}=2 \sqrt{1-4 m_{\sigma}^{2} / s}$ in the center of mass frame. Finally, the average of $s,\langle s\rangle$, is bounded from below, $\langle s\rangle \geq 4 m_{\sigma}^{2}$, where the equality is satisfied when the inflaton quanta are at rest. While we expect them to be non-relativistic at the beginning of reheating, $\langle s\rangle \sim 4 m_{\sigma}^{2}$, we keep the inequality explicitly in the following discussion.

In order for annihilation to be efficient during reheating, the annihilation rate has to be greater than the expansion rate during reheating, $\Gamma_{\mathrm{ann}}>H_{\mathrm{rh}}$. This is satisfied when

$$
\frac{\langle s\rangle}{4 m_{\sigma}}>\frac{10^{-7} \mathrm{GeV}}{\left[F_{2}(v)\right]^{2}}\left(\frac{10^{-6} M_{\mathrm{Pl}}}{H_{\mathrm{rh}}}\right)\left(\frac{10^{32}}{N}\right),
$$

which is a rather weak lower bound on $\langle s\rangle / 4 m_{\sigma}$, which is approximately equal to $m_{\sigma}$ in the non-relativistic limit, for $N \sim 10^{32}$. Therefore, the presence of large- $N$ species makes annihilation very efficient.

Let us compare $\Gamma_{\text {ann }}$ [Eq. (17)] with the decay rate, $\Gamma_{\text {decay }}[$ Eq. (91)]:

$$
\frac{\Gamma_{\text {ann }}}{\Gamma_{\text {decay }}} \gtrsim 24\left(\frac{F_{2}(v) M_{\mathrm{Pl}}}{F_{1}(v)}\right)^{2}\left(\frac{H_{\mathrm{rh}}}{m_{\sigma}}\right)^{2},
$$

where the approximate equality is satisfied when inflaton quanta are non-relativistic. We therefore find that the annihilation channel is not necessarily smaller than the decay channel. It may be more informative to write this result in the following form:

$$
\frac{\Gamma_{\mathrm{ann}}}{\Gamma_{\mathrm{decay}}} \gtrsim 8\left(\frac{F_{2}(v) M_{\mathrm{Pl}}}{F_{1}(v)}\right)^{2} \frac{n_{\sigma}}{M_{\mathrm{Pl}}^{2} m_{\sigma}} .
$$

Thus, there is a critical number density above which the annihilation channel dominates over the decay channel:

$$
n_{\sigma}^{\text {crit }} \equiv \frac{M_{\mathrm{Pl}}^{2} m_{\sigma}}{8}\left(\frac{F_{1}(v)}{F_{2}(v) M_{\mathrm{Pl}}}\right)^{2} .
$$

For $f(\phi)=M_{\mathrm{Pl}}^{2}+\xi\left(\phi^{2}-v^{2}\right)$, for instance, the critical density is given by $n_{\sigma}^{\text {crit }}=$ $M_{\mathrm{Pl}}^{2} m_{\sigma}\left(v / M_{\mathrm{Pl}}\right)^{2}\left[1+6 \xi^{2}\left(v / M_{\mathrm{Pl}}\right)^{2}\right] / 8$.

\section{CONCLUSIONS}

We have studied consistency between Dvali's large- $N$ species solution to the gauge hierarchy problem and inflationary cosmology.

If there exist the large- $N$ species, the inflaton quanta decay or annihilate too efficiently, and reheat the universe too much. We have found that, in order for this scenario to produce successful reheating of the universe, either inflaton mass, vacuum expectation value, or non-minimal gravitational coupling, or all of them, must be fine-tuned to suppress the gravitational decay and annihilation of the inflaton quanta.

We have shown that fine-tuning of an extreme magnitude, Eq. (12), is required by using a widely-studied example. The constraint we have found indeed excludes most of parameter space of the model. This example demonstrates that one must always check whether reheating is successful, whenever their models contain nonminimal coupling, such as $f(\phi) R$ gravity.

One may repeat the same analysis for supergravity inflation models 22, 23, 24] that contain not only nonminimal gravitational coupling (the Kähler potential determines the function $f(\phi))$, but also direct coupling terms in the supergravity frame. However, as supersymmetry alone is able to solve the gauge hierarchy problem (albeit $\mu$ problem still remains), it seems difficult to motivate our having both supersymmetry and large- $N$ species (see, however 25]).

Finally, let us point out the limitation and caveat of our analysis.

The constraints given in this paper are based exclusively upon non-minimal gravitational couplings of inflaton. Therefore, if the non-minimal coupling is totally absent, $f(\phi) \equiv M_{\mathrm{Pl}}^{2}$, or matter fields are conformally coupled to gravity (e.g., massless scalars with $\xi=-1 / 6$, massless fermions, etc), both classically and quantum mechanically, reheating of the universe must be achieved by direct couplings between inflaton and matter fields. Our limits on Dvali's scenario do not apply to such cases. 
We have assumed that the cut-off scale for gravity is much higher than that for the particle physics, $\Lambda$, and thus ignored quantum gravitational effects on the decay rates. It would be interesting to extend our analysis to the case where the cut-off for gravity is also similar to $\Lambda$ [5] or even lower than $\Lambda$ [26].

\section{Acknowledgments}

We would like to thank Arjun Berera, Jim Cline, Gia Dvali, Nemanja Kaloper, and Christos Kokorelis for com- ments on the paper. Y.W. thanks Donghui Jeong and Jun Koda for discussions. E.K. acknowledges support from the Alfred P. Sloan Foundation.
[1] L. Susskind, 1982 Proceedings from the SLAC Summer Institute on Particle Physics: Physics at Very High Energies, (SLAC-R-259, 1982).

[2] S. Weinberg, The Quantum Theory of Fields, Vol. III Supersymmetry, (Cambridge University Press, Cambridge, UK, 2000).

[3] G. Dvali, arXiv:0706.2050 [hep-th]; G. Dvali and M. Redi, arXiv:0710.4344 [hep-th].

[4] G. Veneziano, JHEP 06, 051 (2002).

[5] N. Arkani-Hamed, S. Dimopoulos, and G. Dvali, Phys. Lett. B 429, 263 (1998); Phys. Rev. D 59, 086004 (1999); I. Antoniadis, N. Arkani-Hamed, S. Dimopoulos, and G. Dvali, Phys. Lett. B 436, 257 (1998).

[6] D. Cremades, L.E.Ibanez and F.Marchesano, Nucl. Phys. B 64393 (2002); C. Kokorelis, Nucl. Phys. B 677, 115 (2004).

[7] L. Verde et al., Astrophys. J. Suppl. 148, 195 (2003).

[8] D.N. Spergel et al., Astrophys. J. Suppl. 170, 377 (2007).

[9] L. Page et al., Astrophys. J. Suppl. 170, 335 (2007).

[10] H. Pagels and J.R. Primack, Phys. Rev. Lett. 48, 223 (1982).

[11] G.D. Coughlan, W. Fischler, E.W. Kolb, S. Raby and G.G. Ross, Phys. Lett. B 131, 59 (1983).

[12] J. Ellis, D.V. Nanopoulos, M. Quiros, Phys. Lett. B 174, 176 (1986).

[13] Y. Watanabe and E. Komatsu, Phys. Rev. D 75,
061301(R) (2007), arXiv:gr-qc/0612120

[14] D.S. Salopek, J.R. Bond, and J.M. Bardeen, Phys. Rev. D 40, 1753 (1989).

[15] D.I. Kaiser, Phys. Rev. D 52, 4295 (1995).

[16] T. Futamase and K. Maeda, Phys. Rev. D 39, 399 (1989).

[17] N. Makino and M. Sasaki, Prog. Theor. Phys. 86, 1 (1991).

[18] R. Fakir and W.G. Unruh, Phys. Rev. D 41, 1783 (1990); R. Fakir, S. Habib, and W. Unruh, Astrophys. J. 394, 396 (1992).

[19] J. Hwang and H. Noh, Phys. Rev. Lett. 81, 5274 (1998).

[20] E. Komatsu and T. Futamase, Phys. Rev. D 59, 064029 (1999); 58, 023004 (1998).

[21] S. Tsujikawa and B. Gumjudpai, Phys. Rev. D 69, 123523 (2004).

[22] M. Endo, M. Kawasaki, F. Takahashi, and T.T. Yanagida, Phys. Lett. B 642, 518 (2006).

[23] M. Endo, K. Kadota, K.A. Olive, F. Takahashi, and T.T. Yanagida, JCAP 02, 018 (2007).

[24] M. Endo, F. Takahashi, and T.T. Yanagida, Phys. Rev. D 76, 083509 (2007); arXiv:hep-ph/0701042.

[25] A. Berera and T.W. Kephart, Phys. Rev. Lett. 83, 1084 (1999); Phys. Lett. B 456, 135 (1999).

[26] G. Dvali, G. Gabadadze, M. Kolanovic, and F. Nitti, Phys. Rev. D 65, 024031 (2001). 